\title{
ESTUDIO FITOQUÍMICO DE LAS HOJAS DE Jacaranda caroba (Vell.) DC.
}

\author{
Ingrit Elida Collantes Díaz ${ }^{* a}$, Elfriede Marianne Bacchi ${ }^{\mathrm{b}}$
}

\begin{abstract}
RESUMEN
Jacaranda caroba es un árbol nativo del Brasil, comúnmente usado en la medicina tradicional brasileña. El estudio fitoquímico del residuo clorofórmico del extracto etanólico al $70 \%$ de las hojas, condujo al aislamiento e identificación de los siguientes triterpenos pentacíclicos ácido 3-epi-corosólico (1), ácido 2 $\alpha, 3 \alpha, 19 \alpha$-trihidroxiursano-12-en-28-oico (2), ácido $2 \alpha$-hidroxiursólico (3), ácido maslínico (4), ácido $2 \alpha, 3 \alpha, 23$-trihidroxiursano-12-en-28-oico (5), ácido $2 \alpha, 3 \alpha, 23$-trihidroxioleano-12-en-28-oico (6), ácido $2 \alpha, 3 \beta, 23$-trihidroxioleano12-en-28-oico (7), ácido $2 \alpha, 3 \alpha, 24$-trihidroxiursano-12-en-28-oico (8) y ácido $2 \alpha, 3 \alpha, 24$ trihidroxioleano-12-en-28-oico (9), por comparación de sus espectros de ${ }^{1} \mathrm{H}-\mathrm{RMN}$ y ${ }^{13} \mathrm{C}-\mathrm{RMN}$ con la literatura correspondiente.
\end{abstract}

Palabras clave: Bignonaceae, Jacaranda caroba, triterpenos pentacíclicos.

\begin{abstract}
Jacaranda caroba is a tree native to Brazil, commonly used in Brazilian traditional medicine. Phytochemical study made with the chloroform residue obtained from $70 \%$ ethanol extract of the leaves led to the isolation and identification of pentacyclic triterpenes 3-epi-corosolic acid

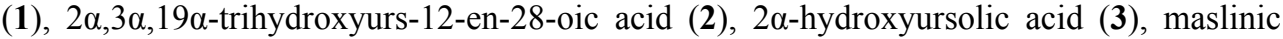
acid (4), 2 $\alpha, 3 \alpha, 23$-trihydroxyurs-12-en-28-oic acid (5), $2 \alpha, 3 \alpha, 23$-trihydroxyolean-12-en-28-

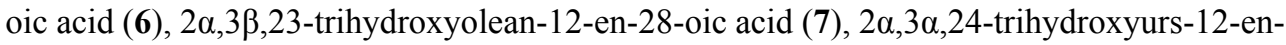
28-oic acid (8) and 2 $\alpha, 3 \alpha, 24$-trihydroxyolean-12-en-28-oic acid (9), identified by literature comparison of their ${ }^{1} \mathrm{H}-\mathrm{NMR}$ and ${ }^{13} \mathrm{C}-\mathrm{NMR}$.
\end{abstract}

Key words: Bignonaceae, Jacaranda caroba, pentacyclic triterpenes.

\section{INTRODUCCIÓN}

La familia Bignonaceae está compuesta por un promedio de 125 géneros y entre 700-800 especies, distribuidas mundialmente en áreas tropicales y subtropicales. El género Jacaranda presenta 49 especies nativas en América del Sur, Central y Caribe, de las cuales 39 son

\footnotetext{
a*Facultad de Ciencias de la Universidad Nacional de Ingeniería, Av. Tupac Amaru 210, Lima, Perú. ingrit_uni@ hotmail.com

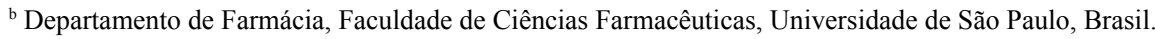


endémicas de Brasil ${ }^{1}$. Jacaranda caroba, en el valle Ribeira del estado de São Paulo es conocida como "caroba", "carobinha"; en otras regiones es conocida también como "camboatá", "camboatá-pequeno", "camboté”, "caroba-do-campo", "caroba-miúda", "caroba muuda", "caroba-do-carrasco". Es considerado un árbol nativo del Brasil, que puede llegar a medir 20 metros de altura, esta especie es encontrada en la selva de la mata atlántica y es de uso ornamental, porque es de rápido crecimiento y fácil de cultivar. Durante mucho tiempo fue usada para hacer carbón, por ser suave y porosa ${ }^{2}$. Según la medicina tradicional brasileña, bañarse con un preparado de las hojas es indicado para infecciones, y beber la infusión de las hojas es usado como depurativo y contra la sífilis; las hojas maceradas en aguardiente es aplicada externamente en heridas y úlceras externas como cicatrizante ${ }^{2}$. Los estudios, desde el punto de vista farmacológico, mencionan la actividad antiúlcera in vivo del extracto etanólico al 70\% de las hojas y el mismo autor, en 1999, demostró que la fracción de cloroformo del extracto hidroalcohólico de las hojas, mostró reducción en las úlceras inducidas por 300mmol/L de ácido clorhídrico en etanol al 60\% (70\% y 100\% de reducción, respectivamente) $)^{3,4}$. Los estudios fitoquímicos, muestran la identificación (en las hojas) de la mezcla de los ácidos ursólico y oleánico en la fracción de diclorometano $\left(\mathrm{CH}_{2} \mathrm{Cl}_{2}\right)$ :acetato de etilo (AcOEt) en la proporción de 97,5:2,5 además de esa mezcla de ácidos, fueron también aislados ácido ursólico y $\alpha$-amirina por Braga ${ }^{5}$. En las hojas fueron identificados 4 isómeros de ácido dicafeoilquínico y los flavonoides isorhamnetina-3-O-ramnósido7,4'-di- $O$-glucósido, isorhamnetina-3- $O$-ramnósido-7/4'-di- $O$-glucósido, quercetina-3$O$-(2-pentosil-6-ramnosil)-glucósido, quercetina-3-O-(2-pentosil)-glucósido, quercetina3-O-(6-ramnosil)-glucósido, quercetina-3-O-glucósido, quercetina-3-O-galactósido, kaemferol-3-O-(6-ramnosil)-glucósido y kaemferol-3-O-glucósido ${ }^{6}$.

En este artículo, abordaremos el aislamiento por cromatografía en camada delgada preparativa y la identificación por $1 \mathrm{H}-\mathrm{y} 13 \mathrm{C}-\mathrm{RMN}$ de nueve triterpenos pentacíclicos presentes en la fracción obtenida por partición con cloroformo del extracto etanol al 70\% de las hojas de $\mathrm{J}$. caroba.

\section{PARTE EXPERIMENTAL}

\section{Recolección de la muestra e identificación botánica}

Fueron colectadas las hojas de J. caroba en el municipio de Leme del estado de São Paulo. El material vegetal fue identificado por la botánica Lucelia Garcez Lohmann, del departamento de botánica de la Universidad de São Paulo y un ejemplar de la especie fue depositado en el herbario SPF de la Universidad de São Paulo con el código de 9.V.2013.

\section{Extracción y aislamiento de metabolitos secundarios}

Las hojas de $J$. caroba fueron secadas en estufa con corriente de aire a $40^{\circ} \mathrm{C}$ por $40 \mathrm{~h}$. Las hojas secas fueron molidas y sometidas a extracción por el método de percolación con etanol al 70\%; el extracto obtenido fue concentrado hasta la completa evaporación del etanol y la fase acuosa fue liofilizada hasta la obtención del extracto bruto de textura pastosa $(39,5 \mathrm{~g})$ de color verde. 
Una parte $(18,99 \mathrm{~g})$ del extracto etanólico al $70 \%$ fue resuspendido en $30 \mathrm{~mL}$ de metanol y $10 \mathrm{~mL}$ de agua para hacer una partición líquido-líquido con los solventes $75 \mathrm{ml}$ de hexano (Hex), $150 \mathrm{ml}$ de cloroformo $\left(\mathrm{CHCl}_{3}\right), 90 \mathrm{ml}$ de acetato de etilo (AcOEt) y metanol-agua, obteniéndose los cuatro residuos de 2,1 g, 2,4 g, 1,09 g, 13,4 g, respectivamente. Una parte del residuo clorofórmico ( $1,8 \mathrm{~g})$ fue sometido a cromatografía en columna (CC), usando como fase estacionaria sílica-gel y como fase móvil la mezcla de solventes Hex, AcOEt y metanol $(\mathrm{MeOH})$ de polaridad creciente. Se obtuvo 68 fracciones, las que fueron sometidas a cromatografía en capa delgada (CCD) analítica en cromatofolios de sílica gel en aluminio de la marca Merck. Los cromatofolios eluidos (en sistemas de eluentes de polaridad creciente de $\mathrm{Hex}: \mathrm{AcOEt}, \mathrm{CHCl}_{3}$ : $\mathrm{AcOEt}$ y $\mathrm{CHCl}_{3}$ : $\left.\mathrm{AcOEt}: \mathrm{MeOH}\right)$ fueron revelados con una solución de ácido sulfúrico (H2SO4) al 20\%, seguida de calentamiento. Se juntaron seis fracciones (4-10, 13-21, 25-30, 32-40, 45-51, 55-61) siguiendo un patrón de semejanza entre ellas por CCD. E1 aislamiento de los metabolitos fue por CCD preparativa en placas de vidrio de $20 \mathrm{x} 20 \mathrm{~cm}$ y $1 \mathrm{~mm}$ de espesor de sílica gel PF 254 (fase estacionaria) de la marca Merck.

De la reunión de la tercera fracción (25-30), se observó una mezcla de compuestos, que fue sometida a CCD preparativa, se usó como fase móvil Hex:AcOEt (2:1), la elución se realizó por tres veces consecutivas y a partir de lo cual se aisló 1 (31,2 mg).

De la fracción 4 (32-40) se obtuvo el compuesto $2(30 \mathrm{mg})$ eluida con $\mathrm{CHCl}_{3}$ :AcOEt $(1: 1)$ sucesivamente. La mezcla de los compuestos 3 y $4(36,9 \mathrm{mg})$ y $\mathbf{5}$ y $\mathbf{6}(72,8 \mathrm{mg})$ fueron aislados de la fracción 5 (45-51) por CCD preparativa eluida con $\mathrm{CHCl}_{3}$ :AcOEt:MeOH (2:2:0,1). Finalmente, eluyendo dos placas por CCD preparativa con la mezcla de los solventes $\mathrm{CHCl}_{3}:$ AcOEt:MeOH $(2: 2: 0,25)$ por tres veces la fracción 6 (55-61), fueron obtenidos 7 (19,8 mg) y la mezcla de 8 y 9 (12,6 mg).

\section{Análisis por Resonancia Magnética Nuclear (RMN).}

Los espectros de Resonancia Magnética Nuclear ${ }^{1} \mathrm{Hy}{ }^{13} \mathrm{C}$ fueron registrados en espectrómetros: Bruker AC-200 (4,69 T) operando a $200 \mathrm{MHz}$ para RMN ${ }^{1} \mathrm{H}$ y $50 \mathrm{MHz}$ para $\mathrm{RMN}{ }^{13} \mathrm{C}$. Espectrómetro de la marca Bruker DRX 300 (7,05T) operando a $300 \mathrm{MHz}$ para RMN ${ }^{1} \mathrm{H}$ y 75 $\mathrm{MHz}$ para $\mathrm{RMN}{ }^{13} \mathrm{C}$. Espectrómetro Varian operando a $500 \mathrm{MHz}$ para $\mathrm{RMN}{ }^{1} \mathrm{H}$ y $125 \mathrm{MHz}$ para ${ }^{13} \mathrm{C}$; los espectros fueron obtenidos en piridina-d5 de la marca Merck.

\section{RESULTADOS Y DISCUSIÓN}

Para realizar el aislamiento de los compuestos presente en este trabajo, ha sido necesario hacer un corte en la lateral de las placas de CCD preparativa para hacer el revelado con H2SO4 20\% seguido de calentamiento, porque los compuestos aislados no revelan frente a la luz ultravioleta a $254 \mathrm{~nm}$.

Compuesto 1.- Fueron aislados $31,2 \mathrm{mg}$ de un sólido crema en cuyo espectro de ${ }^{1} \mathrm{H}-\mathrm{RMN}$ $\left(200 \mathrm{MHz}\right.$, piridina- $d_{5}$ ) se observaron señales como los singuletes a $\delta 0,79(3 \mathrm{H}, \mathrm{H}-24), 0,84$ $(3 \mathrm{H}, \mathrm{H}-25), 0,93(3 \mathrm{H}, \mathrm{H}-26), 1,0(3 \mathrm{H}, \mathrm{H}-23)$ y $1,16(3 \mathrm{H}, \mathrm{H}-27)$. Los singuletes anchos a $\delta$ 
$0,83(3 \mathrm{H}, \mathrm{H}-29)$ y $0,85(3 \mathrm{H}, \mathrm{H}-30)$, cada singulete corresponde a un metilo de la estructura; un doblete a $\delta 2,51(1 \mathrm{H}, J=11,4 \mathrm{~Hz}, \mathrm{H}-18)$; otras señales como $\delta 3,66(1 \mathrm{H}, s, \mathrm{H}-3 \beta), 4,22(1 \mathrm{H}$, $m, \mathrm{H}-2 \beta), 5,35$ singulete ancho $(1 \mathrm{H}, \mathrm{H}-12)$. Los desplazamientos químicos del espectro de ${ }^{13} \mathrm{C}$-RMN de este compuesto presentan las señales como los dobles enlaces de un esqueleto de tipo ursano ${ }^{7}$, que para este caso son 139,22 y 125,35 ppm, la señal característica del C-18 en 53,44 ppm y las señales de los carbonos carbonúlicos C-2 y C-3 $(\mathrm{C}-\mathrm{OH})$ en 65,94 y 79,16 ppm, respectivamente; los 30 desplazamientos químicos están en la tabla 1 y están en concordancia con los publicados en la literatura correspondiente ${ }^{8}$ confirmando que se trata del ácido $2 \alpha, 3 \alpha$-dihidroxiursano-12-en-28-oico también llamado de ácido 3-epi-corosólico (figura 1).
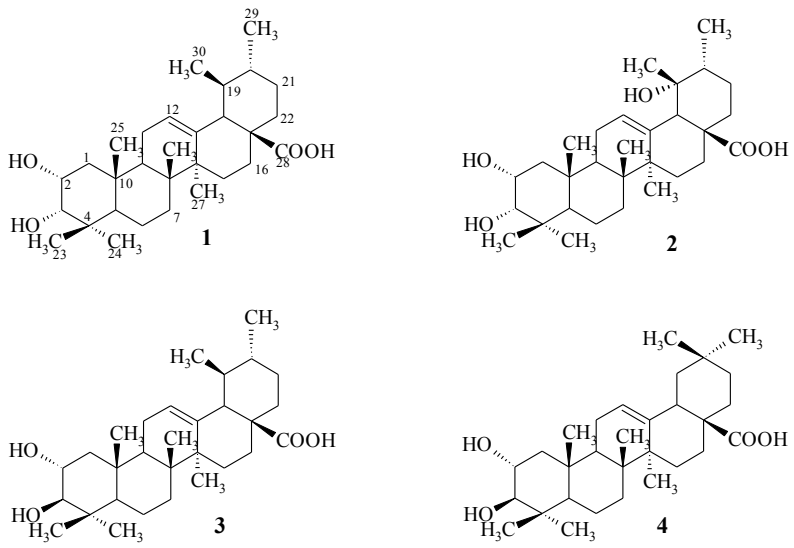

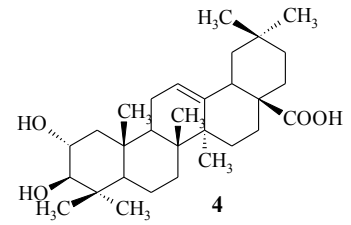

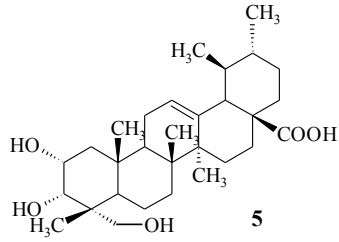

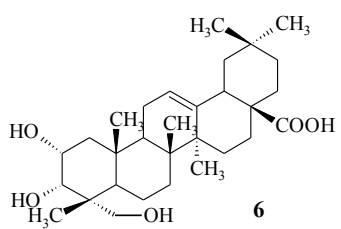<smiles>CC1(C)CCC2(C(=O)O)CCC3C(=CCC4C3(C)CCC3C4(C)C[C@H](O)[C@@H](O)[C@@]3(C)CO)C2C1</smiles>

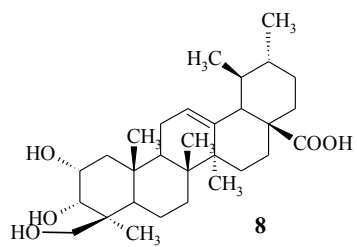

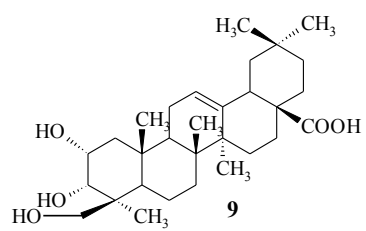

Figura 1. Estructura química de los triterpenos pentacíclicos aislados e identificados en el residuo clorofórmico en el extracto etanólico al $70 \%$ de las hojas de J. caroba. 
Compuesto 2.- Fue aislado $30 \mathrm{mg}$ de un sólido crema cuyo espectro de ${ }^{1} \mathrm{H}-\mathrm{RMN}(300 \mathrm{MHz}$, piridina-d5), presenta señales de los 6 metilos como singuletes a $\delta 0,81(3 \mathrm{H}, \mathrm{s}, \mathrm{H}-24), 0,87$ (3H, s, H-25), 0,98 (3H, s, H-26), 1,15 (3H, s, H-23), 1,30 (3H, s, H-29), 1,52 (3H, s, H-27); un único metilo doblete a $\delta 0,99\left(\mathrm{CH}_{3}, d, J=6,69 \mathrm{~Hz}, \mathrm{H}-30\right)$. También fue observado otras señales como un multiplete a $\delta 2,21$ correspondiente al $\mathrm{H}-15$, un singulete a $\delta 2,93(1 \mathrm{H}$, $s, \mathrm{H}-18$ ), un multiplete a $\delta 2,97$ correspondiente al H-16, 3,65 ppm un singulete ancho $(1 \mathrm{H}, s, \mathrm{H}-3 \beta), 4,19 \mathrm{ppm}$ un doblete ancho $(1 \mathrm{H}, d, J=10,47 \mathrm{~Hz}, \mathrm{H}-2 \beta)$ y un singulete ancho en 5,55 ppm (1H, s, H-12). Los desplazamientos químicos del espectro de ${ }^{13} \mathrm{C}-\mathrm{RMN}$ son característicos de un triterpeno con esqueleto tipo ursano hidroxilado en el C-19 (72,57 ppm), también por las señales de los dobles enlaces en 127,77 (C-12) y 139,87 ppm (C-13), y las señales de los carbonos carbonúlicos C-2 y C-3 en 65,99 y 79,18 ppm, respectivamente, todos los picos que presenta el espectro están en la tabla 1 y la comparación de estas señales con los descritos en la literatura ${ }^{9}$ permitió demostrar que el compuesto 2 es ácido $2 \alpha, 3 \alpha, 19 \alpha-$ trihidroxiursano-12-en-28-oico también llamado como ácido jacarandico (figura 1).

Compuestos 3 y 4.- Fueron aislados 36,9 mg (sólido crema), con el análisis de su espectro de RMN de hidrógeno y de carbono 13, se concluyó que era una mezcla de compuestos que describiremos a continuación; el espectro de ${ }^{1} \mathrm{H}-\mathrm{RMN}(300 \mathrm{MHz}$, piridina-d5) para el ácido $2 \alpha, 3 \beta$-dihidroxiursano-12-en-28-oico también llamado de ácido $2 \alpha$-hidroxiursolico (3) (figura 1) presenta señales como los singuletes anchos de los metilos a $\delta 0,85(3 \mathrm{H}, \mathrm{H}-29)$, $0,86(3 \mathrm{H}, \mathrm{H}-30), 0,88$ (3H, $s, \mathrm{H}-25), 0,89$ (3H, $s, \mathrm{H}-24), 0.96$ (3Hs, H-26), 1,09 (3H, $s, \mathrm{H}-27)$, $1,15(3 \mathrm{H}, s, \mathrm{H}-23)$, otras señales a $\delta 2,52(1 \mathrm{H}, d, J=10,74 \mathrm{~Hz}, \mathrm{H}-18), 3,29(1 \mathrm{H}, d$ ancho, $J=8,85 \mathrm{~Hz}, \mathrm{H}-3 \alpha), 3,98(1 \mathrm{H}, m, \mathrm{H}-2 \beta)$ y 5,35 (1H, $s$ ancho, H-12). Para el ácido $2 \alpha, 3 \beta-$ dihidroxioleano-12-en-28-oico (4) también llamado de ácido maslínico (figura 1) el espectro de ${ }^{1} \mathrm{H}-\mathrm{RMN}(300 \mathrm{MHz}$, piridina-d5) presenta las señales a $\delta 0,83(3 \mathrm{H}, \mathrm{s}, \mathrm{H}-25), 0,86(6 \mathrm{H}, \mathrm{s}$, H-29 y H-30), 0,88 (3H, s, H-26), 0,92 (3H, s, H-23), 0,96 (3H, s, H-24), 1,15 (3H, s, H-27), $3,18(1 \mathrm{H}, \mathrm{d}$ ancho, $\mathrm{J}=10,35 \mathrm{~Hz}, \mathrm{H}-18), 3,29(1 \mathrm{H}, d$ ancho, $J=8,85 \mathrm{~Hz}, \mathrm{H}-3 \alpha), 3,98(1 \mathrm{H}, m$, $\mathrm{H}-2 \beta)$ y $5,35(1 \mathrm{H}, s$ ancho, $\mathrm{H}-12)$. Los desplazamientos químicos del espectro de ${ }^{13} \mathrm{C}-\mathrm{RMN}$ están en la tabla 1 y al ser confrontados con la literatura ${ }^{10}$ se demuestra que es la mezcla de ácido $2 \alpha$-hidroxiursolico (3) y de ácido maslínico (4).

Compuestos 5 y 6 .- Se aisló 78,2 mg de un sólido crema, amorfo; al ser analizado los espectros de ${ }^{1} \mathrm{H}-\mathrm{y}{ }^{13} \mathrm{C}-\mathrm{RMN}$ se ha observado la mezcla de los ácidos $2 \alpha, 3 \alpha, 23$-trihidroxiursano-12-en28-oico (5) y el ácido $2 \alpha, 3 \alpha, 23$-trihidroxioleano-12-en-28-oico (6) (figura 1). El espectro de ${ }^{1} \mathrm{H}-\mathrm{RMN}(200 \mathrm{MHz}$, piridina-d5) los presenta los siguientes desplazamientos químicos de los hidrógeno para 5 como los 4 metilos singuletes de la estructura a $\delta 0,76(3 \mathrm{H}, \mathrm{H}-25)$, $0,88(3 \mathrm{H}, \mathrm{H}-26), 1,02(3 \mathrm{H}, \mathrm{H}-23)$ y $1,09(3 \mathrm{H}, \mathrm{H}-27)$; los dos metilos dobletes a $\delta 0,77(3 \mathrm{H}$, $d, J=6,8 \mathrm{~Hz}, \mathrm{H}-29)$ y $0,89(3 \mathrm{H}, d, J=7,4 \mathrm{~Hz}, \mathrm{H}-30)$. Están presentes también doblete a $\delta 2,49$ con $J=10,6 \mathrm{~Hz}$ del H-18, a $\delta 3,63$ con $\mathrm{J}=10,6 \mathrm{~Hz}$ de uno de los $\mathrm{H}-23$ y a $\delta 3,82$ con $J=10,4$ $\mathrm{Hz}$ corresponde a uno de los $\mathrm{H}-23$, un singulete ancho a $\delta 4,04$ correspondiente al H-3 $\beta$, un doblete ancho a $\delta 4,17$ con $J=9,2$ 
Tabla 1. Desplazamiento químico en piridina-d5 de 13C-RMN (75, 50 y $125 \mathrm{MHz})$ de los compuesto aislados del residuo clorofórmico del extracto etanólico al $70 \%$ de las hojas de J. caroba.

\begin{tabular}{|c|c|c|c|c|c|c|c|c|c|}
\hline C & $1^{1}$ & $2^{2}$ & $3^{2}$ & $4^{2}$ & $5^{1}$ & $6^{1}$ & $7^{2}$ & $8^{3}$ & $9^{3}$ \\
\hline 1 & 42,79 & 42 & 47,95 & 47,66 & 42,4 & & 47,69 & & 43.4 \\
\hline 2 & & & & & & & & & \\
\hline 3 & & & & & & & & & \\
\hline 4 & & & & & & & & & \\
\hline 5 & & & & & & & & & \\
\hline 6 & & & & & & & & & 21 \\
\hline 7 & & & & & & & & &, 00 \\
\hline 8 & & & & & & & & & 0,58 \\
\hline 9 & & & & & & & & & 8,59 \\
\hline 10 & & & & & & & & & 8,92 \\
\hline 11 & 23,6 & & & & & &, 79 & & 4,48 \\
\hline 12 & 5 & & & & & & 22,21 & & 122,8 \\
\hline 13 & 2 & & 139,25 & & & & 145,14 & & 145,22 \\
\hline 14 & 42 & & & & & & 21 & & 2,57 \\
\hline 15 & & & & & & & 32 & & 8,64 \\
\hline 16 & & & & & & & 23,79 & & 4,26 \\
\hline 17 & & & & & & & & & 7,04 \\
\hline 18 & & & & & & & & & 2,57 \\
\hline 19 & & & & & & & & & 55 \\
\hline 20 & & & & & & & & & ,31 \\
\hline 21 & & & & & & & & & ,59 \\
\hline 22 & & & & & & & & & \\
\hline 23 & & & & & & & & & 4,19 \\
\hline 24 & & & & & & & & & 5,59 \\
\hline 25 & & & & & & & & & 7,76 \\
\hline 26 & & & & & & & & & \\
\hline 27 & & & & & & & & & 4,19 \\
\hline 28 & & & & & & & & & \\
\hline 29 & & & & & & & & & \\
\hline 30 & 21,26 & 16,48 & & & 21,21 & $2 J, / J$ & 23,93 & 21,75 & 24,13 \\
\hline
\end{tabular}

${ }^{1}$ Espectro obtenido a $50 \mathrm{MHz},{ }^{2}$ Espectro obtenido a $75 \mathrm{MHz},{ }^{3}$ Espectro obtenido a $125 \mathrm{MHz}$.

$\mathrm{Hz}$ del $\mathrm{H}-2 \beta$ y un singulete ancho en 5,35 ppm del H-12. Los desplazamientos químicos del espectro de ${ }^{13} \mathrm{C}-\mathrm{RMN}$ están en la tabla 1 y al ser comparados con los publicados por Lee ${ }^{11}$, confirman la estructura propuesta (5). Los desplazamientos químicos del espectro ${ }^{1} \mathrm{H}-\mathrm{RMN}$ para la molécula 6 son los singuletes a $\delta 0,76(3 \mathrm{H}, \mathrm{H}-25), 0,79(3 \mathrm{H}, \mathrm{H}-29), 0,88(3 \mathrm{H}, \mathrm{H}-30)$, $0,91(3 \mathrm{H}, \mathrm{H}-26), 1,02(3 \mathrm{H}, \mathrm{H}-23)$ y $1,09(3 \mathrm{H}, \mathrm{H}-27)$, un doblete ancho a $\delta 3,17$ con $J=12,8$ $\mathrm{Hz}$ del $\mathrm{H}-18$, dos dobletes a $\delta 3,63$ y 3,82 con $J=10,6$ y $10,4 \mathrm{~Hz}$ respectivamente, cada señal corresponde a cada uno de los hidrógenos en la posición 23 , un singulete a $\delta 4,04$ del H-3 $\beta$, un doblete a $\delta 4,17$ del $\mathrm{H}-2 \beta$ y un singulete ancho a $\delta 5,35$ del H-12; los desplazamientos químicos del espectro de ${ }^{13} \mathrm{C}-\mathrm{RMN}$ están en la tabla 1 y están de acuerdo con los publicados ${ }^{12}$ para la estructura propuesta 6 (figura 1). 
Compuesto 7 .- Se obtuvo 19,8 mg de un sólido crema cuyo espectro de ${ }^{1} \mathrm{H}-\mathrm{RMN}(300 \mathrm{MHz}$, piridina- $d 5)$, presenta señales como los singuletes a $\delta 0,80(3 \mathrm{H}, \mathrm{H}-25), 0,88(3 \mathrm{H}, \mathrm{H}-29), 0,93$ $(3 \mathrm{H}, \mathrm{H}-30), 0,95(3 \mathrm{H}, \mathrm{H}-26), 0,96(3 \mathrm{H}, \mathrm{H}-23)$ y $1,09(3 \mathrm{H}, \mathrm{H}-27)$, un multiplete a $\delta 3,29(\mathrm{H}-$ 18 ), los dobletes a $\delta 3,61$ y 4,09 (1H cada uno, $d, J=10,44 \mathrm{~Hz}, \mathrm{H}-23)$ y un multiplete a $\delta 5,35$ $(1 \mathrm{H}, \mathrm{H}-12)$. Los desplazamientos químicos del espectro de ${ }^{13} \mathrm{C}-\mathrm{RMN}$ están en la tabla $1 \mathrm{y}$ al comparar con los publicado ${ }^{13}$ se concluye que se trata del ácido $2 \alpha, 3 \beta, 23$-trihidroxioleano12-en-28-oico (7) (figura 1).

Compuestos 8 y 9 .- Fueron aislados 12,6 mg de un sólido crema que al ser analizado los espectros de ${ }^{1} \mathrm{H}-\mathrm{y}{ }^{13} \mathrm{C}-\mathrm{RMN}$, se ha definido que se trata de la mezcla de ácido $2 \alpha, 3 \alpha, 24-$ trihidroxiursano-12-en-28-oico (8) y de ácido 2 $\alpha, 3 \alpha, 24$-trihidroxioleano-12-en-28-oico (9) (figura 1). El espectro de ${ }^{1} \mathrm{H}-\mathrm{RMN}(500 \mathrm{MHz}$, piridina-d5), para ambas estructuras propuestas, la señal correspondiente al $\mathrm{H}-12$ está a $\delta 5,48$; los desplazamientos químicos de los hidrógenos para 8 son la señal del H-3 que está en posición $\beta$, es un singulete a $\delta$ 4,59 , el pico del H-2 $\beta$ es un doblete ancho con $J=9,77 \mathrm{~Hz}$ que está a $\delta 4,46$, uno de los $\mathrm{H}-24$ es un doblete a $\delta 4,12$ con $J=10,74 \mathrm{~Hz}$ y el otro H-24 también es un doblete a $\delta 3,83$ con $J=10,25 \mathrm{~Hz}$, la señal del H-18 es un doblete a $\delta 2,63$ con $\mathrm{J}=11,23 \mathrm{~Hz}$, las señales de los 4 metilos $\left(-\mathrm{CH}_{3}\right)$ son los singuletes a $\delta 0,93(3 \mathrm{H}, \mathrm{H}-25), 1,00(3 \mathrm{H}, \mathrm{H}-26), 1,21(3 \mathrm{H}$, $\mathrm{H}-24), 1,58$ (3H, H-27), los metilos en $\mathrm{H}-29$ y 30 son dos dobletes a $\delta 0,98$ y 1,05 con $J$ de 5,86 y $6,84 \mathrm{~Hz}$, respectivamente. Los desplazamientos químicos del espectro de hidrógeno para 9 son un singulete a $\delta 4,59$ del H-3 $\beta$, un doblete ancho a $\delta 4,46$ de $\mathrm{H}-2 \beta$, dos dobletes a $\delta 4,12$ y 3,83 con $J$ de $10,74 \mathrm{~Hz}$ y $10,25 \mathrm{~Hz}$, respectivamente, que representan los dos $\mathrm{H}-24$, un doblete ancho a $\delta 3,29$ con $J=12,21 \mathrm{~Hz}$ de $\mathrm{H}-18$ y los singuletes a $\delta 0,93(3 \mathrm{H}$, H-25), 0,96 (3H, H-29), 1,00 (3H, H-30), 1,03 (3H, H-26), 1,15 (3H, H-24), 1,69 (3H, $\mathrm{H}-27)$, los desplazamientos químicos del espectro de ${ }^{13} \mathrm{C}-\mathrm{RMN}$ están en la tabla 1 y están en concordancia con los publicados en la literatura correspondiente13.

\section{CONCLUSIONES}

En el presente trabajo fueron aislados e identificados nueve triterpenos pentacíclicos de esqueletos del tipo ursano y oleano, de los cuales 3 de ellos (3-epi-corosólico 1, ácido $2 \alpha, 3 \alpha, 19 \alpha$-trihidroxiursano-12-en-28-oico 2 y ácido $2 \alpha, 3 \beta, 23$-trihidroxioleano-12-en-28oico 7) fueron aislados y 6 están en mezcla de dos ( $2 \alpha$-hidroxiursolico 3 y de ácido maslínico 4; ácido $2 \alpha, 3 \alpha, 23$-trihidroxiursano-12-en-28-oico 5 y el ácido $2 \alpha, 3 \alpha, 23$-trihidroxioleano12-en-28-oico 6 y ácido $2 \alpha, 3 \alpha, 24$-trihidroxiursano-12-en-28-oico 8 y de ácido $2 \alpha, 3 \alpha, 24$ trihidroxioleano-12-en-28-oico 9). Los nueve triterpenos ya fueron identificados y/o aislados en otras plantas, en otras especies del mismo género fue reportado que los compuestos $\mathbf{1 , 2}$ y 3 ya fueron aislados en $J$. caucana ${ }^{14}$ y el compuesto 1 también en J filicifolia $^{15}$. Los nueve compuestos están siendo reportados por primera vez en las hojas de Jacaranda caroba, ya que los triterpenos encontrados anteriormente en la especie en estudio fueron los ácidos ursólico, oleánico y $\alpha$-amirina ${ }^{5}$. 


\section{AGRADECIMIENTO}

Los autores agradecen al Conselho Nacional Pesquisas (CNPq) del gobierno brasileño, por la beca concedida a I.E.C.D. durante el periodo de 2002-2006 para la realización de este trabajo.

\section{REFERENCIAS BIBLIOGRÁFICAS}

1. Gachet MS, Schuhly W. Jacaranda-An ethnopharmacological and phytochemical review. J Ethnopharmacol 2009; 121: 14-27.

2. Di Stasi LC, Hiruma-Lima CA, Plantas medicinais na amazônia e na mata atlântica. Editora UNESP:Botucatu, 2 ed., São Paulo, 2002; pp. 451-452.

3. Bacchi EM. Ação anti-úlcera e cicatrizante de algumas plantas brasileiras. Rev Bras Farmacogn 1986; 1(1): 93-100.

4. Bacchi EM, Rios JPC, Dias TG. Fraction responsible for Jacaranda caroba DC. antiulcer action. In: 2nd IUPAC International Conference on Biodiversity, UFMG,Belo Horizonte, MG. Programme and Abstracts, 1999 p. 173.

5. Braga FC, Valadares YM, Costa MA, Lombardi JA, de Oliveira AB. Estudo fitoquímico de Erythraea centaurium, Jacaranda caroba, Remijia ferruginea e Solanum paniculatum visando identificar marcadores químicos para o fitoterápico Ierobina ${ }^{\circledR}$. Rev Bras Farmacogn 2003; 14: 28-31.

6. Ferreres F, Grosso C, Gil-Izquierdo A, Valentão P, Andrade PB. Phenolic compounds from Jacaranda caroba (Vell) A. DC.: approaches to neurodegenerative disorders. Food Chem Toxicol 2013; 57: 91-98.

7. Militão JSLT, Silva MG de V, de Morais SM, Alencar JW. Contribuição para a determinação estrutural de triterpenos com auxílio de computador. Quim Nova 1996; 19: 121-126.

8. Wen X, Xia J, Cheng K, Zhang L, Zhang P, Liu J, Zhang L, Ni P, Sun H. Pentacyclic triterpenes. Part 5: Synthesis and SAR study of corosolic acid derivatives as inhibitors of glycogen phosphorylases. Bioorg. Med. Chem. Lett. 2007; 17: 5777-5782.

9. Seto T, Tanaka T, Tanaka O, Naruhashi N. $\beta$-Glucosyl esters of $19 \alpha$-Hydroxyursolic acid derivatives in leaves or Rubus species. Phytochem 1984; 23(12), 2829-2834.

10. Taniguchi S, Imayoshi Y, Kobayashi E, Takamatsu Y, Ito H, Hatano T, Sakagami H, Tokuda H, Nishino H, Sugita D, Shimura S, Yoshida T. Production of bioactive triterpenes by Eriobotrya japonica calli. Phytochem 2002; 59: 315-323.

11. Lee IK, Kim DH, Lee SY, Kim KR, Choi SU, Hong JK, Lee JH, Park YH, Lee KR. Triterpenoic acids of Prunella vulgaris var. lilacina and their cytotoxic activities in vitro. Arch. Pharm. Res. 2008; 31(12): 1578-1583.

12. Noel MG, Dayrit FM. Triterpenes in the callus culture of Vitex negundo L. Philipp J Sci 2005; 134(1): 5-19.

13. Li L-M, Pu J-X, Xiao W-L, Sun H-D. Triterpenes from Isodon xerophilus. Chin J Nat Med 2012; 10(4): 307-310.

14. Ogura M, Cordell GA, Farnsworth NR. Potential anticancer agents. IV. Constituents of Jacaranda caucana Pittier. LLoydia 1977; 40(2): 157-168. 
15. Ali RM; Houghton PJ. A new phenolic fatty acid ester with lipoxygenase inhibitory activity from Jacaranda filicifolia. Planta Med. 1999; 65: 455-457. 